\title{
Adrenal insufficiency in a child following unilateral excision of a dual-hormone secreting phaeochromocytoma
}

\author{
Annika Sjoeholm 1 , Cassandra Li ${ }^{1}$, Chaey Leem ${ }^{1}$, Aiden Lee', Maria P Stack ${ }^{2}$, \\ Paul L Hofman ${ }^{3}$ and Benjamin J Wheeler ${ }^{1,4}$ \\ ${ }^{1}$ Department of Women's and Children's Health, University of Otago, Dunedin School of Medicine, PO Box 56, \\ Dunedin 9054, New Zealand \\ ${ }^{2}$ Paediatric Nephrology, Starship Children's Hospital, Auckland, New Zealand \\ ${ }^{3}$ Liggins Institute, University of Auckland, Auckland, New Zealand \\ ${ }^{4}$ Paediatric Endocrinology, Southern District Health Board, Dunedin, New Zealand
}

\author{
Correspondence \\ should be addressed \\ to B J Wheeler \\ Email \\ ben.wheeler@otago.ac.nz
}

\section{Summary}

Phaeochromocytomas are a rare clinical entity, with dual hormone-secreting lesions particularly uncommon, seen in $<1 \%$. ACTH is the most common hormone co-produced, and is potentially lethal if not diagnosed. We present the case of a previously well 10-year-old boy, who presented acutely with a hypertensive crisis and was found to have a unilateral, non-syndromic phaeochromocytoma. Medical stabilization of his hypertension was challenging, and took 3 weeks to achieve, before proceeding to unilateral adrenalectomy. Post-operatively the child experienced severe fatigue and was subsequently confirmed to have adrenal insufficiency. He improved markedly with hydrocortisone replacement therapy, which is ongoing 6 months post-operatively. In retrospect this likely represents unrecognized, sub-clinical ACTH-dependent Cushing's syndrome secondary to an ACTH/or precursor dual-hormone secreting phaeochromocytoma. At follow-up, his hypertension had resolved, there was no biochemical evidence of recurrence of the phaeochromocytoma, and genetic analysis was indicative of a sporadic lesion.

\section{Learning points:}

- Dual hormone secreting phaeochromocytomas with ACTH/or a precursor may cause secondary adrenal insufficiency following surgical removal.

- The concurrent features of Cushing's syndrome can be mild and easily overlooked presenting diagnostic and management pitfalls.

- As concomitant syndromes of hormone excess are rare in phaeochromocytomas; the diagnosis requires a high index of suspicion.

- Serial/diurnal cortisol levels, ACTH measurement +I- low dose dexamethasone suppression (when clinically stable, appropriate adrenergic blockade in place, and well supervised), can all be considered as needed.

\section{Background}

Phaeochromocytomas are rare neuro-ectodermal derived adrenal medullary tumours characterized by disordered production of catecholamines from chromaffin tissue.
They are clinically and functionally heterogenous tumours, and can present a diagnostic challenge. Resistant hypertension and hyperadrenergic spells are classic features, and the main presenting symptoms include: 
headaches, hypertension, nausea and sweating. Initial biochemical testing and diagnosis is suggested with elevated plasma free metanephrines and/or urinary fractionated metanephrines (1) (2). Phaeochromocytomas can be sporadic or as part of a familial syndrome, including type 2 multiple endocrine neoplasia (MEN2), type one neurofibromatosis (NF1), and von Hippel-Lindau disease (VHL) (1) (2).

Ectopic hormone producing phaeochromocytomas are rare, accounting for $1 \%$ of all lesions and demonstrate high phenotypic variability. ACTH appears to be the most common hormone co-produced by phaeochromocytomas, and is a potentially lethal combination (3). In turn, paediatric Cushing's syndrome is also rare and hence presents its own diagnostic challenges. ACTH-independent causes include administration of exogenous glucocorticosteroids, primary adrenal hyperplasia, McCune-Albright syndrome and macronodular adrenal hyperplasia. ACTH-dependent causes include Cushing's disease and the ectopic ACTH-syndrome. Cases of ACTHdependent Cushing's syndrome in association with paediatric phaeochromocytoma are particularly unusual and pose significant challenges for diagnosis and management.

\section{Case presentation}

A 10-year-old boy of mixed Cook Island Maori and Tongan ethnicity presented acutely with hypertensive encephalopathy and generalised seizure activity. He had initially been found unresponsive and then developed focal seizure activity of the left arm and face, with subsequent generalization. Seizure activity was terminated with i.v. midazolam. In the immediate days prior to presentation, the child had experienced photophobia, neck stiffness, headaches, and lethargy.

Further history unmasked an insidious 8-week period of worsening frontal and occipital headaches, associated with anxiety, diaphoresis, palpitations and intermittent vomiting. Episodes were often precipitated by exercise. The child's medical background comprised of atopic dermatitis predominantly localised to the dorsal aspect of his hands and managed with mometasone, locoid and dermol creams. However, he had not used any topical creams in the 6 weeks prior to presenting. Family history consisted of a sister with prior febrile convulsions and maternal hypertension, but was negative for phaeochromocytoma and paraganglionomas.

Initial examination revealed an afebrile, post-ictal child with a Glasgow Coma Scale score of 14, mild photophobia and neck stiffness. He remained hypertensive with a blood pressure of 165/105 (>99th centile for height). There was no hypertensive retinopathy, nil palpable abdominal masses and no dysmorphisms. There were no café-au-lait lesions or other features consistent with NF1, MEN2 or VHL.

\section{Investigation}

An urgent CT head did not demonstrate any intracranial abnormalities or features of raised intracranial pressure. A full septic screen, including lumbar puncture, was unremarkable with normal inflammatory markers and white cell counts. There was mild renal impairment (creatinine of $86 \mu \mathrm{mol} / \mathrm{l}$ ), and urinalysis demonstrated glycosuria. The initial blood glucose level was $11.7 \mathrm{mmol} / 1$. Echocardiography revealed a structurally normal heart with no evidence of co-arctation of the aorta. The serum potassium level was $4.4 \mathrm{mmol} / 1$. Thyroid stimulating hormone and complement levels were within the normal range. A renal ultrasound scan performed to assess for renal artery stenosis, revealed a $2.4 \mathrm{~cm} \times$ $2.1 \mathrm{~cm} \times 1.2 \mathrm{~cm}$ hypoechoic area in keeping with an adrenal mass.

An MRI of the abdomen confirmed a $21 \mathrm{~mm}$ transverse $\times 19 \mathrm{~mm}$ anteroposterior $\times 24 \mathrm{~mm}$ craniocaudal right-sided suprarenal lesion inseparable from the right adrenal gland. The appearances were compatible with a phaeochromocytoma. Nil extension or other intraabdominal pathology was identified (Fig. 1). Analyses of urine and plasma catecholamines and metanephrines were undertaken. Elevated levels confirming the diagnosis of phaeochromocytoma are as follows (all others not mentioned were within normal ranges): plasma normetanephrine $4744 \mathrm{pmol} / 1 \quad(<900)$, urine noradrenaline $5100 \mathrm{nmol} /$ day (0-470), urine normetanephrine $11400 \mathrm{nmol} /$ day (0-2300), and urine vanillyl mandelic acid (VMA) $56 \mu \mathrm{mol} /$ day (0-32).

\section{Treatment}

Pre-operative stabilization of blood pressure over the following 3 weeks was challenging. Prior to knowledge of phaeochromocytoma, he was commenced initially on i.v. hydralazine $(0.15 \mathrm{mg} / \mathrm{kg})$ with minimal impact on his blood pressure. Labetalol (alpha and beta blocker) was given initially i.v. during the hypertensive emergency. Once the mass was identified, alpha-adrenergic blockage was commenced with phenoxybenzamine. Phenoxybenzamine was initiated at $10 \mathrm{mg}(0.2 \mathrm{mg} / \mathrm{kg})$ twice daily, and as tolerated was increased in $10 \mathrm{mg} /$ dose increments 


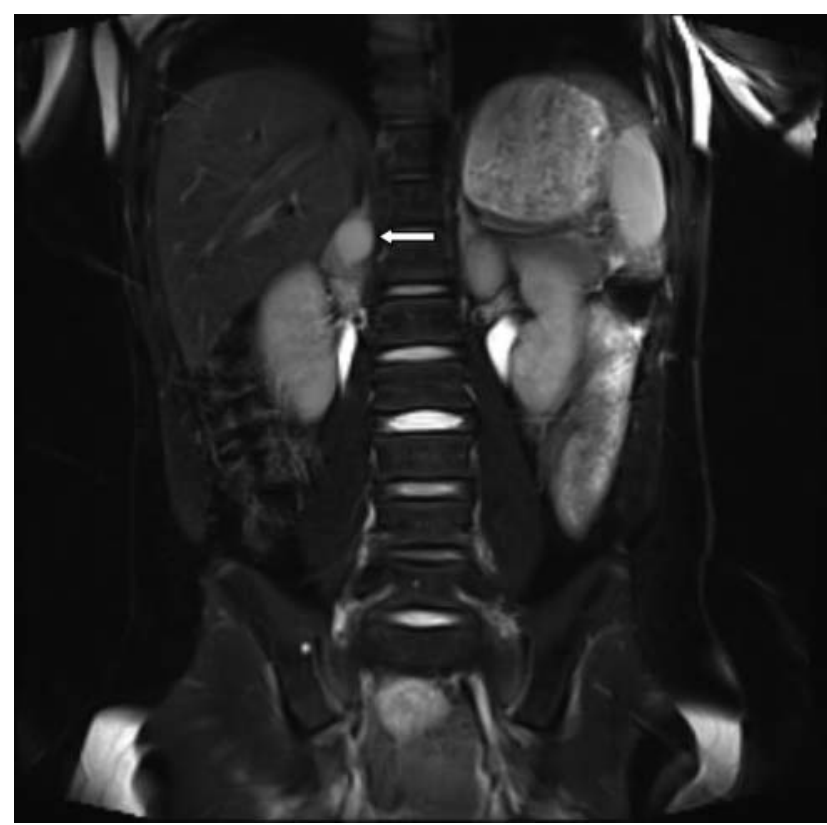

\section{Figure 1}

Coronal T2 weighted MRI of phaeochromocytoma. Arrow indicates tumour.

approximately every 3 days, until he reached a dose of $30 \mathrm{mg}(0.6 \mathrm{mg} / \mathrm{kg})$ twice daily. This dose of phenoxybenzamine provided adequate blood pressure control and adrenergic blockade to safely proceed to surgery. Orthostatic hypotensive symptoms were marked, making mobilization challenging. High salt and fluid intake were required. Labetalol was also added as part of the standard Auckland protocol, to optimize blood pressure control both in the context of the initial hypertensive emergency (BP 180/100 with seizures and headaches) and then later to assist with preoperative preparation in addition to the primary agent, phenoxybenzamine. When using oral labetalol we made incremental dose increases from 0.5 to $2.5 \mathrm{mg} / \mathrm{kg}$ per dose, 8 hourly. It was used during the first week, and once a more stable condition was achieved with excellent alpha blockade via phenoxybenzamine, we switched to the once daily beta blocker, atenolol ( $1 \mathrm{mg} / \mathrm{kg}$ per day), while pending surgery.

The child proceeded to a laparoscopic right-sided adrenalectomy, which was converted to an open procedure due to technical reasons. Gross histology demonstrated a well circumscribed, thinly encapsulated $15 \times 15 \times 15 \mathrm{~mm}$ nodule abutting the adrenal surface. Microscopy demonstrated a tumour composed of small nests of polygonal cells with finely granular eosinophilic cytoplasm and round variably sized nuclei in a network of fine blood vessels. The tumour cells were positive for chromogranin and synaptophysin. The appearances were in keeping with a phaeochromocytoma. Of note, genetic screening for VHL and NF1 were negative, and mutational analysis of the RET proto-oncogene was normal excluding MEN2. Calcium and calcitonin levels were also normal. Maternal plasma metanephrines also returned normal. Succinate dehydrogenase immunostaining was positive with SDHA and SDHB granular cytoplasmic positivity indicating that expression of the SDHA and SDHB was preserved. Subsequent immunohistochemistry was negative, including retrospective ACTH tumour staining.

\section{Outcome and follow-up}

The child spent one night in the Paediatric Intensive Care Unit post-operatively and all antihypertensive medications were ceased with satisfactory blood pressure measurements. The child was discharged home 1 week post-operatively. From this time onwards he experienced severe fatigue, as well as continued headaches and nausea. He was unable to attend school, suffered muscular weakness and difficulty mobilising. Whilst his blood pressure had normalised, on outpatient review postoperatively, examination revealed significantly pigmented gums and palmar creases, tremor and obvious proximal muscle weakness. $250 \mu \mathrm{g}$ i.m. Short synacthen testing demonstrated severe adrenal insufficiency with a baseline $0830 \mathrm{~h}$ cortisol of $<6 \mathrm{nmol} / \mathrm{l}$, and a $45 \mathrm{~min}$ value of $206 \mathrm{nmol} / \mathrm{l}(>550)$. The ACTH level was suppressed at $<0.2 \mathrm{pmol} / 1$ (reference range 1-12). Glucocorticoid replacement therapy was subsequently commenced, and quickly increased to $15 \mathrm{mg} / \mathrm{m}^{2}$ per day to relieve symptoms of severe fatigue. Repeat synacthen testing 6 months later revealed baseline 0830 cortisol of $7 \mathrm{nmol} / \mathrm{l}$; and $60 \mathrm{~min}$ of $400 \mathrm{nmol} / \mathrm{l}$; ACTH was $6.3 \mathrm{pmol} / \mathrm{l}$ (1-12), all suggesting partial but not yet complete recovery of the hypothalamic-pituitary-adrenal (HPA) axis. Follow up phaeochromocytoma screening has been so far normal.

\section{Discussion}

We have described a rare case of adrenal insufficiency following unilateral adrenalectomy of a phaeochromocytoma in a 10-year-old child. In the absence of any other explanation, we propose that the phaeochromocytoma had likely dual-hormone secreting capacity, and that the child had unrecognized subclinical Cushing's syndrome pre-operatively. Suppression of the HPA axis from this ectopic source of ACTH, or ACTH-precursor, likely resulted in the subsequent post-operative presentation of 
symptomatic adrenal insufficiency. To date, only two paediatric cases have been reported in the English literature with this occurrence, a 15-year-old girl (4) and an 11-month-old boy (5). These cases presented predominantly with symptoms of Cushing's syndrome and were only found to have phaeochromocytomas on histology. In contrast, our case presented with classic features of phaeochromocytoma and catecholamine excess (2), and only in retrospect had features of subclinical Cushing's syndrome, that likely impacted upon pre-operative blood pressure stabilisation.

Phaeochromocytomas themselves are a rare clinical entity in paediatric endocrinology, and similar to our report, present at an average age of 11 years, with a slight predominance in males. Around $10-20 \%$ of the estimated incidence of $\leq 0.3$ cases per million people will present in childhood, and of these only $\sim 1 \%$ will be dual-hormone secreting, most commonly with ACTH or an ACTH precursor (2). However, even mild and subclinical Cushing's syndrome in combination with a phaeochromocytoma is a potentially lethal combination. Making it vital that clinicians are aware of this possibility, as only a high index of clinical suspicion will lead to appropriate and timely diagnosis. The evaluation of Cushing's in this scenario is as per standard practise, with one essential caveat. Dexamethasone suppression testing (DST), can rarely precipitate a steroid induced hypertensive emergency, or catecholaminergic crisis, and has led to death in one instance following high dose DST (6). While this has never been reported for the low dose DST (6), it seems prudent that this aspect of evaluation is undertaken only when the patient is clinically stable, well supervised, and has appropriate adrenergic blockade in place.

In addition, the current diagnostic criteria for ACTHsecreting phaeochromocytomas includes (7):

i) Clinical and laboratory evidence of hypercortisolism.

ii) Elevated plasma ACTH level.

iii) Evidence of a phaeochromocytoma by urinary catecholamines, metanephrines, and VMA, or MRI evidence of an adrenal mass with a bright $\mathrm{T} 2$ signal.

iv) Resolution of symptoms-signs of adrenocorticoid and catecholamine excess after unilateral adrenalectomy.

v) Rapid normalization of plasma ACTH levels after adrenalectomy.

In this report, the first criterion is not obviously fulfilled. A pre-operative random cortisol level was within the normal range ( $206 \mathrm{nmol} / \mathrm{l}$ at $0730 \mathrm{~h}$ ), however Friedeman et al. argue that a single determination of eucortisolism is insufficient to exclude Cushing's syndrome. In mild, or subclinical Cushing's syndrome hypercortisolism may be intermittent or short-term, necessitating two or more measures of cortisol, and at two different time periods (8). In retrospect, at follow-up, the child's body composition was leaner and his facial features were thinner, raising the likelihood of previously unrecognized Cushing's syndrome. As regards the second criterion, a pre-operative ACTH level was not assessed, however the clinical finding of gum hyperpigmentation at the postoperative follow-up may be a residual indicator of previously elevated ACTH levels. Criterion three was clearly fulfilled as outlined in the case report with grossly elevated plasma and urine catecholamines.

The fourth criterion is fulfilled upon careful examination of the child's presenting signs and symptoms. In a review of 24 adult patients with ACTH-producing phaeochromocytomas, most of the patients presented with severe symptoms of Cushing's syndrome, hypertension, hyperglycaemia and severe hypokalaemia (9). In accordance with these features, our case presented with hypertension, hyperglycaemia and glycosuria, all of which resolved following unilateral adrenalectomy. Hypokalaemia in the ectopic ACTH syndrome is associated with a higher rate of cortisol production. Given the proposition that our child had mild or subclinical Cushing's syndrome, and therefore possibly only intermittent or short-term hypercortisolism, this could explain why he did not present with hypokalaemia. Paediatric Cushing's syndrome is often unrecognized by clinicians, perhaps due to the rarity and variable clinical phenotype, particularly in mild cases. The child's symptoms of catecholamine excess subsided post-adrenalectomy. Similarly, his hyperglycaemia, glycosuria and alteration in body composition, which suggested subclinical Cushing's syndrome, also subsided post-operatively.

The final criterion is met through normalization of ACTH levels post-operatively. The first ACTH level was taken post-operatively, and was low/suppressed at $<0.2 \mathrm{pmol} / \mathrm{l}$ suggestive of HPA-axis suppression. By 6 months post-operatively the child's ACTH level had normalized to $6.3 \mathrm{pmol} / \mathrm{l}$. The child has required ongoing glucocorticoid replacement post-operatively. This is in keeping with previous cases in which successful surgical removal of an ectopic ACTH-producing tumour unmasks secondary adrenal insufficiency, such that nearly all patients require glucocorticoid replacement therapy until the HPA-axis recovers (10). 
Retrospective ACTH-staining of the excised phaeochromocytoma was negative, however this does not rule out an ACTH/ACTH precursor-producing phaeochromocytoma. Cassarino et al. (11) described a case of an ACTHproducing phaeochromocytoma with false-negative immunohistochemistry. Molecular techniques revealed that the tumour had high POMC mRNA, and high expression of PC1 (prohormone convertase which processes POMC into ACTH), demonstrating that the phaeochromocytoma synthesized and secreted ACTH despite negative ACTH immunostaining. Similarly all of the hypothalamo-pituitary axis peptides ACTH, POMC and CRH have been demonstrated in phaeochromocytomas with concomitant Cushing's syndrome, such that negative ACTH-staining is accounted for by production of ACTH precursors. It is also possible that high peptide secretion of ACTH could result in negative staining due to ACTH not being stored within the synthesizing cells (11). Chen et al. (7) noted that in one of four cases there was negative ACTH-staining, but insufficient evidence to rule out biologically active ACTH secretion. It is also possible that if the tumour is in a quiescent phase at the time of surgery then this could also result in negative ACTH staining (8).

Administration of exogenous glucocorticoids is a possible cause of ACTH-independent Cushing's syndrome. We argue that the child's use of topical steroids is an unlikely cause of his presentation with post-operative adrenal insufficiency. Whilst there are reports in the literature to support HPA-axis suppression with topical corticosteroid use, these are largely in infants and patients using grade one potent topical steroids over a large body surface area and on a daily basis. In this child's case, mometasone fumorate was used sparingly over the dorsum of his hands on average 5-7 days per month, and interestingly he had not used any treatment in the 6 weeks prior to presenting. In addition, Schlessinger $e t$ al. (12) demonstrated no HPA-axis suppression following a 2 -week course of daily super high potency $0.1 \%$ fluocinomide cream.

In conclusion we have presented a very rare case of secondary adrenal insufficiency in a 10-year-old child, following unilateral phaeochromocytoma excision. A dual-hormone secreting tumour appears the most likely explanation. This case highlights the importance of a high index of clinical suspicion as regards dual-hormone secreting lesions. We propose that this should be considered possible in all patients, particularly those with atypical features or difficult to manage hypertension, and consideration for further evaluation be undertaken, using serial/diurnal cortisol levels and ACTH measurement initially, $+/-$ low dose dexamethasone suppression (when clinically stable, appropriate adrenergic blockade in place, and well supervised, due to the risk of steroid inducing hypertensive emergencies). This high index of suspicion is particularly important in the paediatric age group, as Cushing's syndrome is often mild and overlooked.

Declaration of interest

The authors declare that there is no conflict of interest that could be perceived as prejudicing the impartiality of the research reported.

\section{Funding}

This research did not receive any specific grant from any funding agency in the public, commercial or not-for-profit sector.

\section{Patient consent}

Written informed consent has been obtained from the patient (and the patient's guardian) for publication of the submitted article and accompanying images.

\section{Author contribution statement}

A Sjoeholm, C Li, C Leem, and A Lee produced the first draft of the manuscript. A Sjoeholm and B J Wheeler coordinated the manuscript through subsequent editing and submission. M P Stack, P L Hofman, and B J Wheeler provided clinical care to the patient, as well as working on the manuscript. B J Wheeler conceived and supervised the manuscript. All authors reviewed and approved the final version.

\section{References}

1 Van Berkel A, Lenders JW \& Timmers HJ 2014 Diagnosis of endocrine disease: biochemical diagnosis of phaeochromocytoma and paraganglioma. European Journal of Endocrinology/European Federation of Endocrine Societies 170 R109-R119. (doi:10.1530/EJE-13-0882)

2 Waguespack SG, Rich T, Grubbs E, Ying AK, Perrier ND, Ayala-Ramirez M \& Jimenez C 2010 A current review of the etiology, diagnosis, and treatment of pediatric pheochromocytoma and paraganglioma. Journal of Clinical Endocrinology and Metabolism 95 2023-2037. (doi:10.1210/jc.2009-2830)

3 Kirkby-Bott J, Brunaud L, Mathonet M, Hamoir E, Kraimps JL, Tresallet C, Amar L, Rault A, Henry JF \& Carnaille B 2012 Ectopic hormone-secreting pheochromocytoma: a francophone observational study. World Journal of Surgery 36 1382-1388. (doi:10.1007/s00268012-1488-1)

4 Li XG, Zhang DX, Li X, Cui XG, Xu DF, Li Y, Gao Y, Yin L \& Ren JZ 2012 Adrenocorticotropic hormone-producing pheochromocytoma: a case report and review of the literature. Chinese Medical Journal 125 1193-1196.

5 Kumar M, Kumar V, Talukdar B, Mohta A \& Khurana N 2010 Cushing syndrome in an infant due to cortisol secreting adrenal pheochromocytoma: a rare association. Journal of Pediatric Endocrinology \& Metabolism 23 621-625. (doi:10.1515/jpem.2010.102)

6 Barrett C, van Uum SH \& Lenders JW 2015 Risk of catecholaminergic crisis following glucocorticoid administration in patients with an 
Endocrinology

Diabetes \& Metabolism

CASE REPORT
A Sjoeholm and others
Dual-hormone secreting phaeochromocytoma
ID: 15-0041; June 2015

DOI: 10.1530/EDM-15-0041 adrenal mass: a literature review. Clinical Endocrinology. In press. (doi:10.1111/cen.12813)

7 Chen H, Doppman JL, Chrousos GP, Norton JA, Nieman LK \& Udelsman R 1995 Adrenocorticotropic hormone-secreting pheochromocytomas: the exception to the rule. Surgery 118 988-994; discussion 94-95. (doi:10.1016/S0039-6060(05)80104-7)

8 Friedman TC, Ghods DE, Shahinian HK, Zachery L, Shayesteh N, Seasholtz S, Zuckerbraun E, Lee ML \& McCutcheon IE 2010 High prevalence of normal tests assessing hypercortisolism in subjects with mild and episodic Cushing's syndrome suggests that the paradigm for diagnosis and exclusion of Cushing's syndrome requires multiple testing. Hormon- und Stoffwechselforschung = Hormones et Métabolisme 42 874-881. (doi:10.1055/s-0030-1263128)

9 Nijhoff MF, Dekkers OM, Vleming LJ, Smit JW, Romijn JA \& Pereira AM 2009 ACTH-producing pheochromocytoma: clinical considerations and concise review of the literature. European Journal of Internal Medicine 20 682-685. (doi:10.1016/j.ejim.2009.08.002)

10 Biller BM, Grossman AB, Stewart PM, Melmed S, Bertagna X, Bertherat J, Buchfelder M, Colao A, Hermus AR, Hofland LJ et al. 2008 Treatment of adrenocorticotropin-dependent Cushing's syndrome: a consensus statement. Journal of Clinical Endocrinology and Metabolism 93 2454-2462. (doi:10.1210/jc.2007-2734)

11 Cassarino MF, Ambrogio AG, Pagliardini L, De Martin M, Barresi V, Cavagnini F \& Pecori Giraldi F 2012 ACTH-secreting pheochromocytoma with false-negative ACTH immunohistochemistry. Endocrine Pathology 23 191-195. (doi:10.1007/s12022-012-9212-0)

12 Schlessinger J, Miller B, Gilbert RD, Plott RT \& Vanos Study Group 2006 An open-label adrenal suppression study of $0.1 \%$ fluocinonide cream in pediatric patients with atopic dermatitis. Archives of Dermatology 142 1568-1572. (doi:10.1001/archderm.142.12.1568)

Received in final form 11 May 2015

Accepted 15 May 2015 